\title{
High resolution magnetic resonance imaging of the anterior visual pathway in patients with optic neuropathies using fast spin echo and phased array local coils
}

\author{
A Gass, G J Barker, D MacManus, M Sanders, P Riordan-Eva, P S Tofts, J Thorpe, \\ W I McDonald, I F Moseley, D H Miller
}

\begin{abstract}
High resolution MRI of the anterior visual pathways was evaluated using frequency selective fat suppressed fast spin echo (FSE) sequences in conjunction with phased array local coils in patients with optic neuropathies. Fifteen normal controls and 57 patients were examined. Coronal T2 weighted fat suppressed FSE images were obtained in 11 minutes with an in plane resolution of $0.39 \times 0.39 \mathrm{~mm}$. The optic nerve and its sheath containing CSF were clearly differentiated. Central retinal vessels were often visible. In demyelinating optic neuritis and in anterior ischaemic optic neuropathy high signal within the nerve was readily delineated. Meningiomas and gliomas involving the optic nerve were precisely visualised both in the orbit and intracranially. Extrinsic compression of the optic nerves was readily visualised in carotid artery ectasia and dysthyroid eye disease. Enlarged subarachnoid spaces around the optic nerves were demonstrated in benign intracranial hypertension. High resolution MRI of the anterior visual pathway represents an advance in the diagnosis and management of patients presenting with optic neuropathy.
\end{abstract}

(F Neurol Neurosurg Psychiatry 1995;58:562-569)

Key words: magnetic resonance imaging; fast spin echo; fat suppression; phased array coil; optic neuropathy

Each optic nerve consists of about 1 million myelinated nerve fibres; the optic nervesheath complex measures 4 to $6 \mathrm{~mm}$ in diameter and its length from the globe to the optic chiasm varies between 45 and $55 \mathrm{~mm} .{ }^{1}$ Successful imaging of the optic nerve is difficult as it is a small, often tortuous, and mobile structure surrounded by fat within the orbit, by bone within the optic canal, and by CSF. Intracranially it lies very close to the carotid arteries. There are many causes of optic neuropathy, and optimal management is dependent on making a correct diagnosis. Neuroradiological procedures are especially important in the diagnosis of extrinsic com- pressive lesions and primary tumours of the optic nerve; CT has been the preferred investigation for orbital pathology as conventional MBI sequences are impeded by high signal from orbital fat. Magnetic resonance imaging is more effective in the region of the optic canal and the intracranial portion of the nerve where bone hardening artefacts limit the value of CT. Even the combination of high resolution CT and conventional MRI, however, is not always satisfactory, and subtle lesions exerting little or no mass effect are not infrequently missed. ${ }^{2}$ Short tau inversion recovery (STIR) and frequency selective fat suppressed spin echo sequences have proved useful in reducing orbital fat signal, but only relatively low resolution images are possible within clinically acceptable time constraints. ${ }^{3-7}$ Using a conventional surface coil placed in front of the eyes, signal to noise ratio (SNR) is improved within the orbit, but decreased in the optic chiasm and adjacent regions. ${ }^{8} 9$ Two recent technical developments, however-phased array local coils and the fast spin echo (FSE) sequence-now make it possible to obtain high resolution images of the anterior visual pathways in an acceptable time. ${ }^{10-13} \mathrm{We}$ report our findings of the use of a combination of fat suppressed FSE and phased array coils in an "optimised" imaging protocol for the anterior visual pathways. The advantages and potential of such an imaging approach are outlined.

\section{Materials and methods}

MAGNETIC RESONANCE IMAGING

Imaging was performed on a 1.5T Signa (General Electric, Milwaukee, USA) MRI unit. All subjects were studied in the coronal plane using an "optimised" (see appendix) mildly T2 weighted FSE sequence with frequency selective fat suppression with a pair of 3 inch circular coils. ${ }^{56}$ Imaging parameters were: TR3250/Tef68, ETL 16, NEX = 6, field of view $20 \mathrm{~cm}, 512 \times 512$ matrix, rectangular field of view, $3 \mathrm{~mm}$ interleaved contiguous slices, 11 minutes. In 28 patients fat suppressed T1 weighted spin echo or gradient echo sequences were used for imaging before and after injection of gadolinium-DTPA $(0.1 \mathrm{mmol} / \mathrm{kg})$, using a field of view of $16-20 \mathrm{~cm}$ and a matrix of $256 \times 256$. In six patients with optic nerve tumours additional 
axial images were obtained using the same sequences.

The phased array local coils were positioned symmetrically over the temples, such that optimum proximity to the optic nerves was obtained. Patients were asked to avoid excessive eye movement and to keep their eyes closed to avoid globe motion artefact. After axial localising scans at the level of the optic nerve entering the globe, coronal slices were adjusted to the angle needed for symmetric imaging of the two nerves.

All films were reviewed by an experienced neuroradiologist (IFM). In normal controls, patients with benign intracranial hypertension, optic neuritis, optic nerve sheath meningiomas, and optic nerve gliomas, structured reporting schemes were used. The scans of patients with benign intracranial hypertension, optic neuritis, anterior ischaemic optic neuropathy, and of normal controls were mixed and reported by IFM without knowledge of the clinical details.

\section{PATIENTS AND CONTROLS}

We studied 57 patients with optic neuropathies and 15 normal controls. Patients were recruited from the National Hospitals of Neurology and Neurosurgery and the physicians' clinic and orbital clinic of Moorfields Eye Hospital. Informed consent was obtained in writing from all subjects. The research protocol had prior approval of the National Hospital's joint medical ethics committee.

Normal controls

Fifteen normal controls (age range 20-41) were studied.

\section{Optic neuritis}

Fourteen patients (age range 19-41) with a clinical diagnosis of demyelinating optic neuritis, supported by abnormal visual evoked potentials, were studied after three days to six months (mean $2 \cdot 4$ months) of visual loss. ${ }^{14}$

Anterior ischaemic optic neuropathy

Four patients with the clinical diagnosis of anterior ischaemic optic neuropathy were studied within four weeks of the onset of symptoms and again after six months. ${ }^{15}$

\section{Benign intracranial hypertension}

Benign intracranial hypertension is a condition of obscure aetiology; patients were included in this category if they had increased intracranial pressure, papilloedema, and normal CSF constituents in the absence of an intracranial mass or hydrocephalus; 13 patients with benign intracranial hypertension were examined. ${ }^{16}$

\section{Meningiomas involving the optic nerve}

Meningiomas may arise directly from the orbital dural sheath or the optic nerve may be compressed by tumour arising intracranially from the clinoid or tuberculum sellae. ${ }^{17} 18 \mathrm{We}$ studied 12 patients with meningiomas involving the optic nerve. Histological confirmation was available in six patients.

\section{Optic nerve glioma}

Optic nerve gliomas often have a benign histological appearance and although they may occur at any age, most patients present in the first decade..$^{19}{ }^{19} \mathrm{We}$ studied five patients with optic nerve gliomas. Histological confirmation was available in two patients.

\section{Central retinal artery occlusion}

Two patients with sudden onset visual field defects due to central retinal artery occlusion were studied within four weeks of visual loss and again after six months. ${ }^{20}$

\section{Leber's hereditary optic neuropathy}

Two patients with visual loss due to Leber's hereditary optic neuropathy, confirmed by genetic testing, were studied 11 months and three years after onset of visual loss. ${ }^{21-23}$

\section{Dysthyroid eye disease}

We studied two patients with symptomatic dysthyroid eye disease. ${ }^{24} 25$

\section{Carotid ectasia}

One patient with optic nerve compression due to ectatic carotid arteries was studied. ${ }^{26}$

\section{Tobacco-alcohol neuropathy}

Two patients with a clinical diagnosis of tobacco-alcohol amblyopia were studied. ${ }^{27} 28$

\section{Results}

Local coil positioning was well tolerated by all subjects. Motion artifact from globe movement was minimal and image quality was consistently good. Fat suppression was achieved in all patients without major artefact or variation of contrast.

\section{Normal controls}

Visualisation of the optic nerve and its internal structure was provided. The differentiation of the nerve from surrounding CSF within its dural sheath was possible in the anterior orbit in all studies. The width of the optic nerve sheath was symmetric in normal controls but varied between subjects with CSF visible on two to five slices in the orbit. It narrowed progressively towards the orbital apex with no CSF signal seen in the optic canal. None of the controls showed changes in morphology or signal suggesting a lesion in the optic nerve. High signal from the central retinal vessels was visible in 10 of 15 controls. The image quality of the chiasmal area was similar to that of the intraorbital portion of the optic nerve.

\section{Optic neuritis}

Hyperintense lesions were seen within all symptomatic nerves. Lesion length varied from one to 12 slices (3-36 mm). The intraorbital portion of the optic nerve was involved in 13, the intracanalicular portion in four, and the intracranial portion in five studies. In long lesions, visible on more than five slices, signal change was visible in both the intracanalicular and intracranial portions. The coronal 


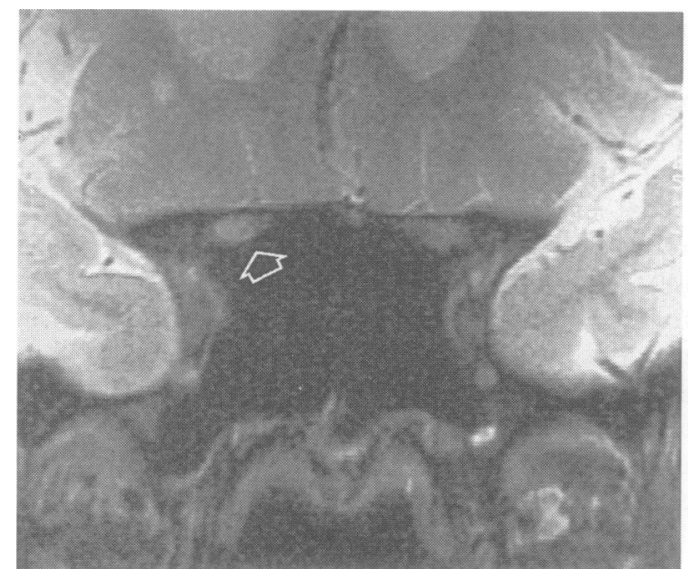

A

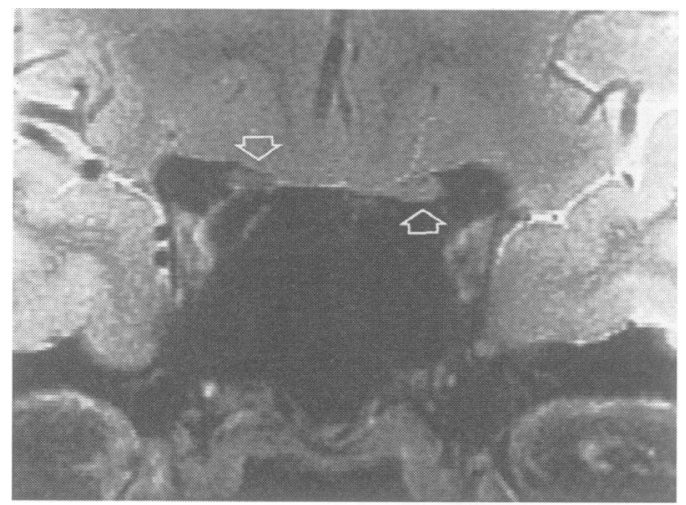

C

imaging plane showed smaller degrees of signal abnormality at the edge of lesions and in two patients partial cross sectional involvement of the optic nerve was seen. In the acute stage, swelling of the nerve attributable to oedema, with no CSF visible surrounding the nerve, was seen in three patients (fig $1 \mathrm{~A}-\mathrm{C}$ ).

\section{Benign intracranial hypertension}

The ring of CSF surrounding the optic nerve was demonstrated throughout the orbit and

Figure 2 Coronal T2 weighted FSE fat suppression image of the anterior orbit in a patient with benign intracranial hypertension. Asymmetric widening of the subarachnoid space with a wider, enlarged CSF space on the left can be seen (arrow). Signal characteristics and size of the optic nerves are normal. Asymmetry of disc swelling was seen on fundoscopy with pronounced papilloedema on the left.

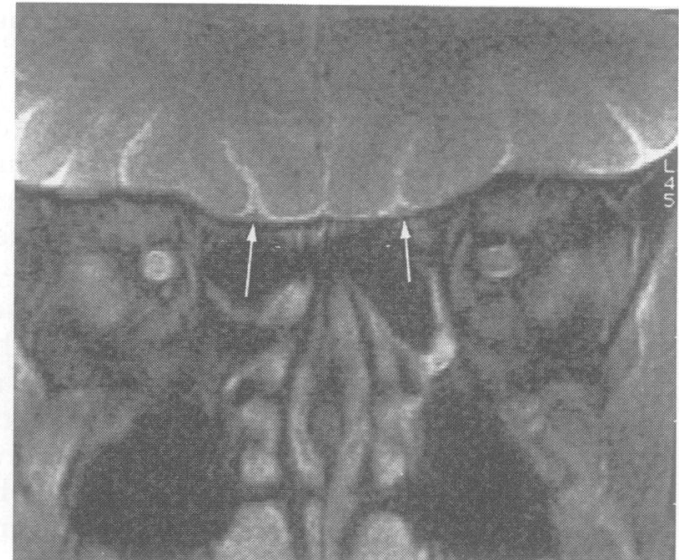

B

Figure 1 Coronal T2 weighted FSE fat suppression images showing high signal lesions in different locations in three patients with optic neuritis $(A)$ Lesion of the intracanalicular portion of the right optic nerve (arrow); (B) high signal lesion of the posterior orbital portion of the right optic nerve, a fine ring of CSF surrounding the optic nerve can be seen bilaterally; the olfactory tracts are seen as triangular structures (arrowed); (C) high signal lesion of the left optic nerve intracranially (arrow up); normal signal intensity similar to cerebral white matter is shown in the right optic nerve (arrow down).

into the optic canal (on eight to 11 slices). In four patients with asymmetric disc swelling on fundoscopy, the asymmetry of papilloedema correlated with the width of the optic nerve sheath seen on MRI (figure 2).

Meningiomas involving the optic nerve

Two cases showed mainly intraorbital tumour growth, 10 of 12 showed mainly intracranial tumour masses. The tumour tissue was clearly identified and delineated from surrounding structures in all patients. Gadoliniumenhanced imaging and T2 weighted sequences demonstrated the presence or absence of tumour growth in the optic canal and dural abnormalities intraorbitally. The topographic relation of nerve and tumour was clearly demonstrated intracranially. Tumour surrounding the optic nerve in the optic canal or intraorbitally was shown in eight patients. Gadolinium enhanced T1 weighted images gave a target like appearance in six patients; in two patients enhancement of the optic nerve was also demonstrated (fig 3).

\section{Optic nerve gliomas}

The appearance of the gliomas was that of a swollen, fusiform, enlarged optic nerve. Intrinsic optic nerve signal was abnormal, but to different degrees in different parts of the lesion, quite unlike the rather homogeneous hyperintensity seen in demyelinating optic neuritis lesions. Three patients showed an enlarged optic canal on the side of the lesion. The nerve kinked intraorbitally and in four patients there was no subarachnoid CSF space identifiable. Chiasmal involvement was demonstrated in one patient. The T2 weighted images showed intrinsic signal 


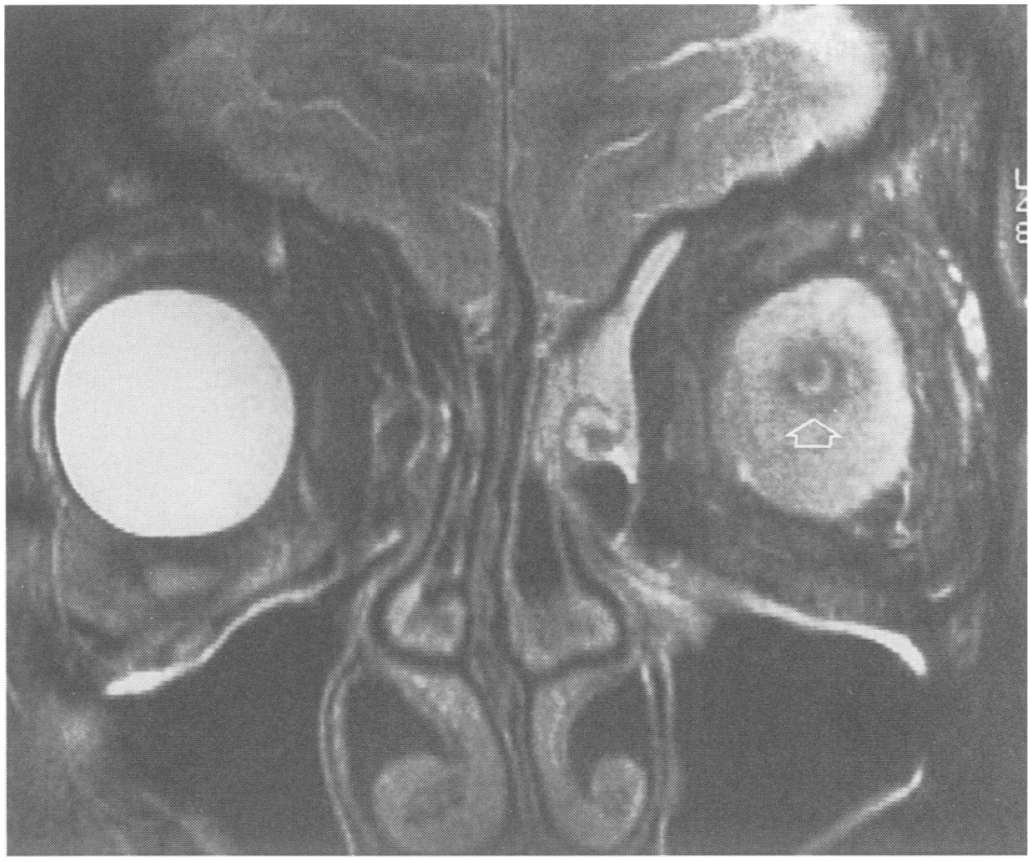

Figure 3 Coronal T2 weighted FSE fat suppression image of a patient with an intraorbital optic nerve sheath meningioma showing a "target" like formation (arrow) with an optic nerve in the centre of white matter intensity, bright CSF around it, and the tumour mass surrounding this concentrically.

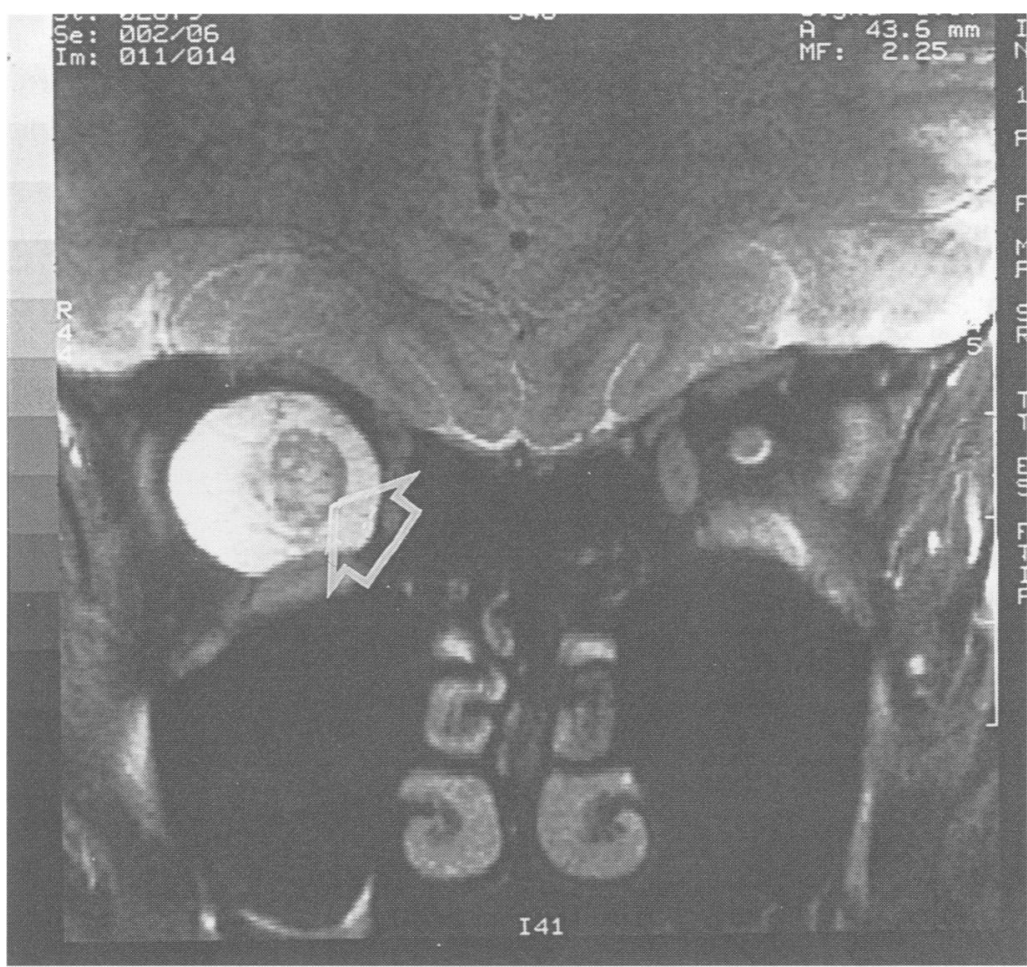

Figure 4 Coronal T2 weighted FSE fat suppression image showing a right optic nerve glioma with a grossly enlarged optic nerve with heterogeneous intrinsic signal from the tumour (arrow). High signal surrounds the tumour representing CSF or mucoid degeneration, which causes compression of the extraocular muscles; the left optic nerve-sheath complex shows normal signal characteristics. abnormalities of the chiasm in one case in which its size and shape were normal (fig 4).

Anterior ischaemic optic neuropathy

No signal abnormality could be demonstrated at presentation, but after six months the affected nerves showed long high signal lesions involving at least five slices, which were of normal size (fig $5 \mathrm{~A}, \mathrm{~B}$ ).

\section{Dysthyroid eye disease}

Images clearly showed the involved extraocular muscles and to what extent orbital fat and extraocular muscles contributed to soft tissue enlargement. No intrinsic optic nerve lesions were seen (fig $6 \mathrm{~A}, \mathrm{~B}$ )

Leber's hereditary optic neuropathy

Bright signal was seen in the optic nerves of two virtually blind patients. The optic nervesheath complex was small and no CSF was visible surrounding the nerves.

\section{Tobacco-alcohol neuropathy}

No signal abnormality was visible in the optic nerves intracranially or in the optic-sheath complexes.

\section{Ectatic carotid arteries}

Prominent enlarged carotid arteries were demonstrated intracranially and were associated with compression of the optic nerves,

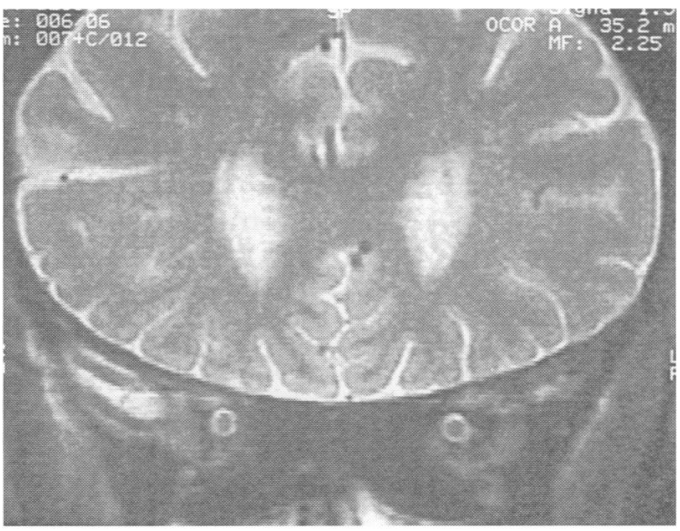

A

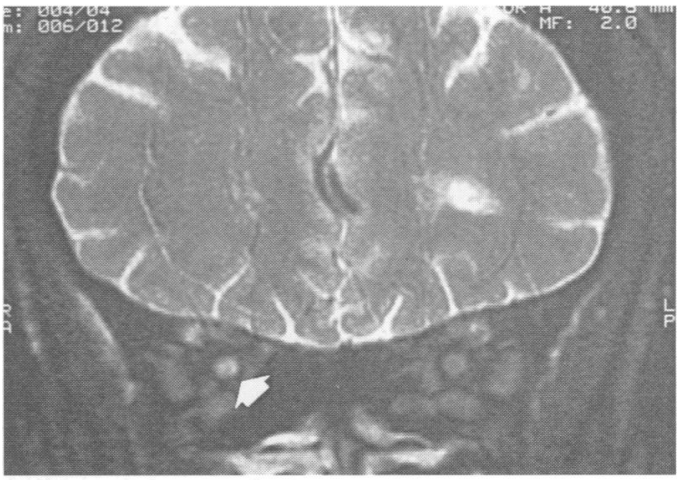

B

Figure 5 Coronal T2 weighted FSE fat suppression images in a patient with anterior ischaemic optic neuropathy. $(A)$ Showing no abnormality in the right optic nerve 13 days after symptom onset; $(B)$ on the follow up examination after six months a high signal lesion is demonstrated (arrow). 
which were displaced by the arteries, and indentation of brain parenchyma. The optic nerves and chiasm were extremely small and thin (fig 7).

\section{Discussion}

By combining the FSE sequence with phased array coils we have shown that high resolution, high signal to noise ratio images of the anterior visual pathway, from the globe to the chiasm, can be collected in just over $10 \mathrm{~min}$ utes. The ability to provide fine anatomical detail and images that are sensitive to pathological changes offers an important advance in the differential diagnosis of optic neuropathies, and the monitoring of their treatment, and in some instances should provide new insights into the underlying pathophysiological mechanisms. High anatomical resolution offers a more confident interpretation of the small area of the optic nerve-sheath complex.

A problem with small conventional surface coils was the signal drop off in the optic canal and chiasm. ${ }^{29} \mathrm{With}$ lateral positioning, the signal from phased array coils decreased towards the centre of the head, but the signal to noise ratio was adequate in all parts of the anterior visual pathway. By magnifying the central portion of the image slightly, the extremely high signal areas of subcutaneous fat and grey matter in the lateral temporal lobes immediately under the coils were excluded, making it easier to window the images optimally. This was the only postprocessing requirement in our study. Homogeneity correction can be achieved by division of phantom image, but did not offer additional information and did not help in the description and interpretation of normal anatomy or pathological findings. ${ }^{30}$

In demyelinating optic neuritis, although diagnosis on clinical grounds, supported by visual evoked potentials, is usually straightforward, occasional difficulty may arise in making a distinction from acute anterior ischaemic optic neuropathy. ${ }^{31}$ Optic nerve MRI in such instances should be useful in the acute phase, when signal abnormality in the nerve will occur in demyelinating but not in ischaemic optic neuropathies. In optic neuritis we could demonstrate high signal lesions in all symptomatic nerves, which underlines the high sensitivity of this imaging approach to the inflammatory-demyelinating optic nerve lesion.

A particular value in accurately characterising the symptomatic lesion in demyelinating optic neuritis is the opportunity to elucidate the pathophysiological mechanisms of demyelinating CNS disease. This is possible in the optic nerve, where a unique correlation of the evolving clinical, electrophysiological (visual evoked potentials), and pathological (MRI) events can be monitored. On lower resolution scans a correlation between acute visual loss in optic neuritis and gadolinium enhancement, the enhancement indicating the presence of acute inflammation, has been

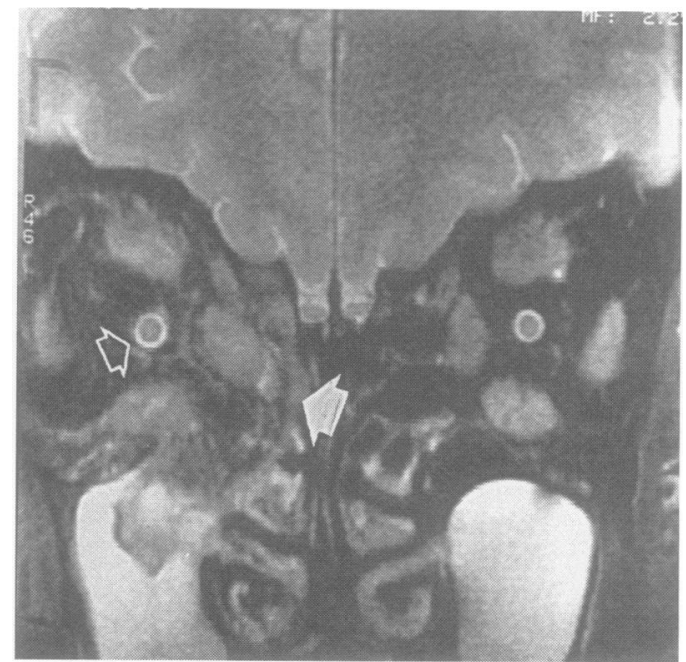

A

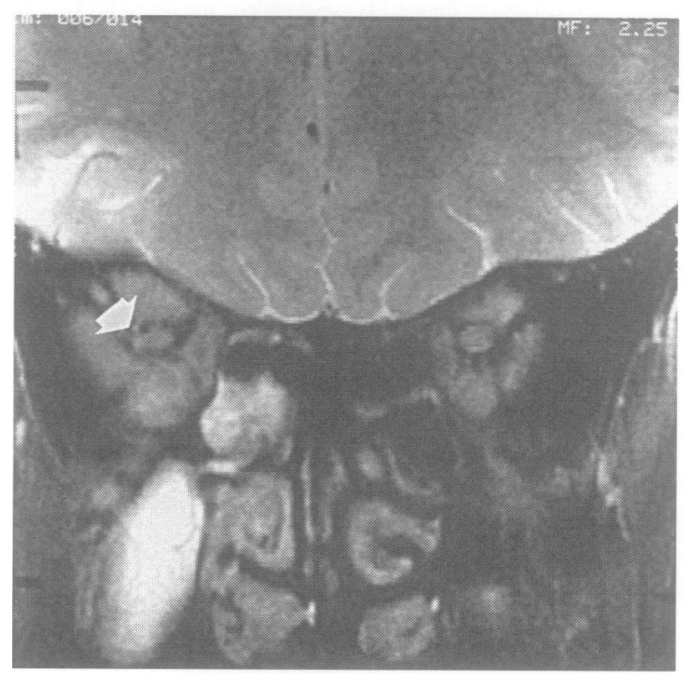

B

Figure 6 Coronal T2 weighted FSE fat suppression images of a patient with dysthyroid eye disease after surgical decompression of the medial wall of the right orbit. $(A)$ On the midorbital image displacement and enlargement of intraorbital soft tissues is shown (solid arrow). The optic nerve-sheath complex has normal signal characteristics (open arrow). (B) In the posterior orbit flattening of the right optic nerve (solid arrow) is seen by grossly enlarged extraocular muscles.

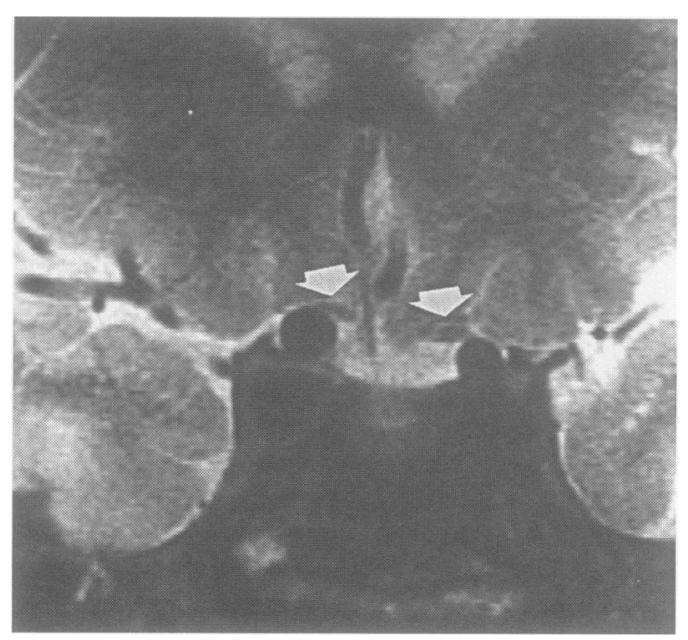

Figure 7 Coronal T2 weighted FSE fat suppression image showing indentation of the gyrus rectus by an enlarged right carotid artery. Thin. flattened optic nerves (arrows) more so on the right can be seen medially adjacent to the carotid arteries. 
found. ${ }^{14}$ In the future, the combination of high resolution imaging with quantitative $M R$ approaches thought to be sensitive to demyelination and axonal loss (for example, magnetisation transfer imaging ${ }^{32} 33$ and $\mathrm{T} 2$ magnetisation decay curve analysis ${ }^{34}$ ) should give further insights into the mechanisms of relapse, recovery, and failure to recover.

The features we demonstrated in benign intracranial hypertension were diagnostically useful and cannot be reliably shown with either lower resolution MRI or high resolution CT. ${ }^{35}$ The increased size of the subarachnoid space correlates with raised intracranial pressure, provided that the optic nerves themselves are of normal size. As medical treatment of benign intracranial hypertension is largely empirical and surgical intervention such as fenestration of the optic nerve sheath is sometimes advocated, high resolution MRI may well be a useful objective tool for showing that the sheaths are dilated and may safely be incised, and for monitoring treatment. ${ }^{36}$

Meningiomas involving the optic nerve are often diagnosed relatively late. Radiotherapy may provide visual stabilisation. ${ }^{17}$ If surgical intervention is to be undertaken, complete resection of the tumour is needed to provide any chance of a permanent cure. ${ }^{17}$ Intraorbitally growing tumour may be inoperable because of the inevitable risk of damage to the optic nerve and complete removal of intracranial tumour has often been impossible as the topographic relation of the tumour to the carotid arteries is so intimate. ${ }^{37}$ In focal intracranially arising meningiomas the presence of intracanalicular tumour extension and the extent of intracranial tumour is important in identifying patients at the greatest risk of losing vision. We could demonstrate both these features. In two patients surgery was performed after demonstration that intracranial tumour caused compression of the optic nerve but had not yet entered the optic canal. Whether such presurgical imaging information may be of prognostic value regarding the long term outcome remains to be seen. Exact topographic information about the degree of encasement of the carotid arteries was clearly provided in all cases with intracranial tumour growth. High resolution MRI should also become an important follow up investigation to monitor the speed and location of tumour growth in patients treated conservatively or with radiotherapy and to detect recurrence in those treated with surgery. Tumour spread across the sphenoid bone towards the opposite side into the periforaminal area can also be clearly seen with high resolution T2 weighted and postgadolinium enhanced scans.

Optic nerve gliomas are usually slow growing tumours, and are therefore often treated conservatively. The rate of tumour growth and involvement of the optic chiasm and retrochiasmal structures are of prognostic importance. ${ }^{19}$ Therefore, clinical assessment and MRI are the mainstays of monitoring tumour progression and extent. High resolution MRI is perfectly suited to this purpose.
Optic nerve compression is an important cause of visual loss in dysthyroid eye disease and may require surgical decompression, systemic corticosteroid treatment, or radiotherapy. ${ }^{32}$ Magnetic resonance imaging offers an objective measure of orbital soft tissue swelling (extraocular muscles, intraorbital fat), and optic nerve compression. The response to surgical decompression or medical treatment can be monitored accurately with a T2 weighted FSE study.

High signal lesions in the optic nerve have been previously demonstrated in Leber's hereditary optic neuropathy, but the small size of the optic nerve-sheath complex along with the lack of CSF around the nerve is a new finding. ${ }^{39}$ The specificity of this pattern needs to be assessed by further study of other conditions that are associated with chronic optic nerve atrophy.

The compression of the optic nerves produced by enlarged intracranial carotid arteries has not been previously demonstrated by MRI. Even though this is a rarely diagnosed disorder, coronal high resolution MRI may help to identify vascular compression causing visual loss, especially in the elderly population.

Finally, the value of gadolinium enhancement has been already noted in optic neuritis and in optic nerve tumours. ${ }^{14}{ }^{40-42}$ Postgadolinium images of either conventional $(256 \times 128)$ or high $(512 \times 512)$ resolution are a very useful complement to the data obtained with high resolution $\mathrm{T} 2$ weighted FSE images in the differential diagnosis of optic neuropathies.

We are grateful for the provision of fast spin echo and phased array local coils by General Electric, Milwaukee, USA. We thank Mr John Wright for kindly agreeing to the study of his patients.

The NMR Research Group is funded by a generous grant from the Multiple Sclerosis Society of Great Britain and Northern Ireland, and supported by the Brain Research Trust and the Medical Research Council.

\section{Appendix}

TECHNICAL NOTE: OPTIMISATION OF IMAGING PROTOCOL

The anterior visual pathways are difficult to image with a clinically acceptable resolution and signal to noise ratio (SNR), in a clinically acceptable time, by conventional spin echo based techniques. We therefore performed a stepwise optimisation of an imaging protocol in normal controls, to investigate the utility of phased array coils (to increase SNR) and FSE (to increase speed) in this anatomical area.

Phased array coils

Phased array coils combine the high SNR of a local coil with the field of view (FOV) of a much larger coil. 910 They are particularly useful for imaging large structures close to the surface, or for simultaneously imaging several small structures. In this study a pair of 3 inch circular coils (provided by General Electric Medical Systems, Milwaukee, USA), originally intended for temporomandibular joint imaging, were placed over the temples to determine whether this provided higher SNR than the standard head coil for images of the anterior visual pathways from the globe to the optic chiasm.

SNR comparison quadrature head coil: phased array coils We performed an SNR comparison of phased array local coils and the standard head coil using identical 
fat suppressed FSE imaging parameters. For any local coil the received signal, and thus the SNR, varies from point to point; for phased array coils the situation is further complicated by the processing used to combine the data from each coil into a single image, which also varies from point to point. We therefore defined SNR as the ratio of mean signal over a small area to the mean background intensity in an adjacent "zero" signal area. A mean signal from the optic nerves themselves was measured in both optic nerves on all slices. The mean background signal (representing the noise) was measured in the sinuses. In all cases the same regions were used on both the head coil and phased array coil scans. With the phased array coil, a $26-39 \%$ SNR gain was seen relative to the head coil along the length of the optic nerve.

\section{Fat suppressed $S E$}

Assuming that the relaxation properties of the nerve are similar to those of cerebral white matter a fat suppressed spin echo sequence (SE2500/70) allows 10 slices ( $5 \mathrm{~mm}$ thickness, $1 \mathrm{~mm}$ gap) to be collected in a single acquisition. A matrix of $256 \times 128$ and two averages (NEX) takes just under 11 minutes and with a $16 \mathrm{~cm}$ FOV yields an in plane resolution of $1.25 \times$ $0.625 \mathrm{~mm}$. Fat suppression can be achieved by using frequency selective presaturation pulses. ${ }^{4}$ This provides good fat suppression T2 weighted spin echo images and allows the nerve to be clearly distinguished from its surroundings, but the sequence does not provide enough resolution to see details of the nerve structure.

\section{Fast spin echo (FSE)}

Fast spin echo is based on the rapid acquisition with relaxation enhancement (RARE) sequence first described by Hennig et al. ${ }^{13}$ In essence, the technique acquires a multiecho train (typically four, eight, 16 or more echoes) following each excitation. A different phase encoding pulse is applied in conjunction with each echo, which allows multiple lines of image data to be collected alter each excitation. Thus the time required is radically reduced compared with a spin echo sequence at the expense of slightly less slices per acquisition. An FSE 2500/95 (echo train length (ETL) 16) with fat suppression and the same in plane resolution as the above SE sequence takes only 45 seconds, and covers seven slices in a single acquisition. Increasing the matrix size to $256 \times 256$, and setting the number of excitations (NEX) $=4$ increases the scan time to two minutes 45 seconds, and a $512 \times 512$ matrix, again with $\mathrm{NEX}=4$, takes 11 minutes for the two acquisitions necessary to collect sufficient slices to study the whole optic nerve. Resolution in the $512 \times$ 512 image is very good, but the $5 \mathrm{~mm}$ slice thickness leads to blurring of the edges and large partial volume effects if the nerve is not aligned perpendicular to the slice. The SNR of the images is also too low to be clinically acceptable.

\section{Rectangular FOV}

Rectangular FOV, which collects only the central $50 \%$ of an image, reduces imaging time by a factor of two. A requirement for the technique to be successful, however, is that very little signal should be present outside this reduced field of view, as it will wrap back into the image producing unacceptable artefacts. The natural drop off of the 3 inch coils, combined with superior and inferior spatial presaturation pulses, fulfils this condition when imaging the optic nerves. The extra time gained by using a rectangular FOV can be used to increase the number of aquisitions, which in turn allows the slice thickness to be reduced to $3 \mathrm{~mm}$. This gives more isotropic resolution, reducing partial volume effects, but reduces the SNR of the resulting images still further.

\section{An "optimal" combination}

The SNR lost by moving to $512 \times 512$ images and $3 \mathrm{~mm}$ slices (with a $16 \mathrm{~cm}$ FOV) can be partially compensated for by the use of the phased array coils, but the average $30 \%$ gain in SNR is not in itself enough to produce clinically acceptable images. Slightly increasing the FOV and increasing the number of averages recovers more SNR, and increasing the TR (to reduce $\mathrm{T} 1$ weighting) and reducing the TE slightly (to decrease T2 weighting) gives a further increase in SNR. This leads to an "optimal" sequence: FSE $3250 / 68(\mathrm{ETL}=16, \mathrm{NEX}=6$, FATSAT, rectangular FOV, $20 \mathrm{~cm}$ FOV, $512 \times 512$ matrix, $3 \mathrm{~mm}$ slices) which gives 14 slices in 11 minutes with the added bonus that the two acquisitions required allow contiguous interleaved slices to be collected.

1 Kupfer C, Chumbley L, Downer J. Quantitative histology of optic nerve, optic tract and lateral geniculate ganglionucleus of man. $\mathcal{f}$ Anat 1967;101:393-401.

2 Moseley IF. Diagnostic imaging in visual loss: a problem oriented approach. Imaging 1992;4:151-5.

3 Johnson G, Miller DH, MacManus DG, et al. STIR sequences in NMR imaging of the optic nerve. sequences in NMR imaging
Neuroradiology 1987;29:238-45.

4 Miller DH, Newton MR, van der Poel JC, et al. Magnetic resonance imaging of the optic nerve in optic neuritis. Neurology 1988;38:175-9.

5 Simon J, Szumowski J, Tottermann S, et al. Fat suppression MRI of the orbit. AfNR Am $\mathcal{F}$ Neuroradiol 1988; 9:961-8

6 Hendrix LE, Kneeland JB, Haughton VM, et al. MR imaging of optic nerve lesions: value of gadopentate dimeglumine and fat suppression techniques. $A \Im N R$ Am f Neuroradiol 1990;11:749-54.

7 Miller DH, MacManus DG, Bartlett PA, Kapoor R, Morrissey SP, Moseley IF. Detection of optic nerve lesions in optic neuritis using frequency selective fat satulesions in optic neuritis using frequency selective
ration sequences. Neuroradiology 1993;35:156-8.

8 Atlas SW, Bilaniuk LT, Zimmermanu RA, et al. Orbit: initial experience with surface coil spin echo MR imaging at 1.5 T. Radiology 1987;164:501-9.

9 Atlas SW, Grossman RI, Savino PJ, et al. Surface coil of orbital pseudotumor. AfNR Am $\mathcal{F}$ Neuroradiol 1987;8: 141-6.

10 Roemer PB, Edelstein WA, Hayes CE, et al. The NMR phased array. Magn Reson Med 1990;16:192-225.

11 Hayes CE, Roemer PB. Noise correlations in data simultaneously aquired from multiple surface coil arrays. Magn Reson Med 1990;16:181-91.

12 Constable RT, Anderson AW, Zhong J, et al. Factors influencing contrast in fast spin-echo $M R$ imaging. Magn Reson Imaging 1992;10:497-511.

13 Hennig J, Naureth A, Friedburg H. RARE imaging: a fast imaging method for clinical MR. Magn Reson Med 1986; 3:823-33

14 Youl BD, Turano G, Miller DH, et al. The pathophysiology of optic neuritis. Brain 1991;114:2437-50.

15 Boghen DR, Glaser JS. Ischaemic optic neuropathy: the clinical profile and natural history. Brain 1975;75 689-99.

16 Wall M, George D. Idiopathic intracranial hypertension. Brain 1991;114:155-80.

17 Wright JE, Call NB, Liaricos S. Primary optic nerve meningioma. $\mathrm{Br} F$ Ophthalmol 1980;64:553-8.

$18 \mathrm{McDonald}$ WI. The symptomatology of tumours of the anterior visual pathways. Can $\mathscr{f}$ Neurol Sci 1982;9: 381-90.

19 Wright JE, McDonald WI, Call NB. Management of optic nerve gliomas. Br f Ophthalmol 1980;64:545-52.

20 Zimmermann LE. Embolism of the central retinal artery. Arch Ophthalmol 1965;73:822-6.

21 Hancock. Hereditary optic atrophy (Lebers disease). Royal London Ophthalmological Hospital Reports 1908;17: 167-77

22 Wallace DC, Singh G, Lott MT, et al. Mitochondrial DNA mutation associated with Leber's hereditary optic neuropathy. Science 1988;242:1427-8.

23 Holt JI, Miller DH, Harding AE. Genetic heterogeneity and mitochondrial DNA heteroplasmy in Leber's hereditary optic neuropathy. $\mathcal{F}$ Med Genet 1989;26 739-43.

24 Neigel JM, Rootman J, Belkin RI et al. Dysthyroid optic neuropathy. The crowded orbital apex syndrome. Ophthalmology 1988;95:1515-8.

25 Trokel SL, Jakobiec FA. Correlation of CT scanning and pathologic features of ophthalmic Graves' disease. Ophthalmology $1981 ; 88: 553-64$.

26 Unsold R, Seeger W. Compressive optic nerve lesions at the optic canal. 1st ed. Berlin: Springer, 1989:245-8.

27 Samples JR, Younge BR. Tobacco-alcohol amblyopia. f Clin Neuroophthalmol 1981;1:213-6.

28 Kermode A, Miller DH. MRI of Tobacco-alcohol amblyopia. $\mathcal{F}$ Neurol Neurosurg Psychiatry 1989;52:1447.
.

29 Atlas SW. Magnetic Resonance imaging of the orbit: current status. Magn Reson $Q$ 1989;5:39-96.

30 Tofts PS, Barker GJ, Simmons A, McManus DG, Gass A Miller DH. Correction of nonuniformity of the spine and optic nerve from fixed receiver-only surface coils at 1.5T. F Comp Assist Tomog 1994 (in press).

31 Rizzo IF, Lessell S. Optic neuritis and ischemic optic neuropathy: overlapping clinical profiles. Arch Opthalmo 1991;109:1668-72. 
32 Wolff SD, Balaban RS: Magnetisation transfer contrast (MTC) and tissue water proton relaxation in vivo. $M a g$ Res Med 1989;10:135-44.

33 Dousset V, Grossman R, Ramer KN, Sclmall MD, Young LH, Gonzalez-Scarano F, et al. Experimental allergic encephalomyelitis and multiple sclerosis: lesion characencephalomyelitis and multiple sclerosis: lesion charac1992;182:483-91.

34 Barnes D, Munro PMG, Youl BD, et al. The longstanding MS lesion. A quantitative MRI and electron microing MS lesion. A quantitative MRI and

35 Hamed LM, Tse DT, Glaser JS, et al. Neuroimaging of the optic nerve after fenestration for management of pseudotumor cerebri. Arch Ophthalmol 1992;110:636-9.

36 Kelman SE, Heaps R, Wolf A, et al. Optic nerve decompression surgery improves visual function in patients with pseudotumor cerebri. Neurosurgery 1992;30:391-5.

37 Al-Melty O. Clinoidal meningiomas. $\mathcal{f}$ Neurosurg 1990;73: $840-9$.
38 Bartalena L, Marcocci C, Chiomoto C, et al. Orbital cobalt irradiation combined with systemic corticosteroids in Graves' ophthalmopathy: comparison with systemic corticosteroids alone. 7 Clin Endocrinol Metab 1983;56:1139-44.

39 Kermode AG, Moseley IF, Kendall BE, Miller DH, MacManus DG, McDonald WI. Magnetic resonance imaging in Leber's optic neuropathy. F Neurol Neurosurg imaging in Leber's optic neuropathy. F Neurol Neurosurg
Psychiatry 1989;52:671-4.

40 Guy J, Mancuso A, Quisling RD, et al. Gd-DTPAenhanced MRI in optic neuropathies. Ophthalmology 990;97:592-600.

41 Lindblom B, Truwit C, Hoyt WF. Optic nerve meningioma: definition of intraorbital, intracanalicular, and intracranial components with magnetic resonance imaging. Ophthalmology 1992;99:560-6.

42 Eidelberg D, Newton MR, Johnson G, et al. Chronic unilateral optic neuropathy: a magnetic resonance study. Ann Neurol 1988;24:3-11. 